\title{
Optimization of Essential Parameters in Green Sand Process to Minimize Persisting Casting Defects Using Taguchi Approach
}

\author{
S.N. Aloni \\ Mechanical Engineering, DMIETR, Wardha, India
}

Received 14 December 2018; Accepted 14 September 2019

\begin{abstract}
The main objective of the work presented in this paper is the identification of most influential parameters of green sand casting process that is responsible for the occurrences of the defects which are persisting in complex gray iron components. The shrinkage porosity, blowholes, sand inclusion and low hardness are the defects which are persisting in cylinder head castings produced in gray iron by a green sand casting process. In pursuing for the identification of most influential parameters for respective defects, Taguchi's approach to parameter design is proposed. In the first stage of the study, 19 potential parameters associated with principal operations are selected and experiments are performed as per the plan given by the suitable Taguchi array, L36 OA. In the second stage optimal levels of the parameters are determined from the signal-to-noise ratio calculations. Analysis of variance (ANOVA) is subsequently performed to identify most influential parameters. Confirmation run of experiments is performed with optimal settings of parameters to verify the results. Outcome of confirmation experiments indicated that persisting defects in cylinder head castings produced by a green sand process are sufficiently reduced.
\end{abstract}

Keywords: Green sand process, persisting casting defects, process parameters, Taguchi orthogonal array, analysis of variance: ANOVA

\section{Introduction}

The clay bonded sand, commonly known as green sand, is typically bonded with Bentonite (clay) and water to mould the sand. Other common sand casting processes used for the production of casting include CO2, No-Bake, and Shell Sand process depending on the type of sand used for production of mould. The choice of particular sand casting process depends upon the factors like the size and intricacy of the components, as well as the volume of production [1]. Wide range of gray iron castings required in automotive and other engineering applications is produced in medium \& largescale foundries by a green sand process. The main aim of the foundry industries is to produce quality castings with no rejection. Also, in the present era of global competition, manufacturing industries has bound to take the challenge that is demand for high quality product with reduced lead-time [2].In the green sand casting process number of parameters are involved that are associated with main operations performed during casting production. The quality of castings produced in green sand casting process, is always a result of a proper combination and settings of these parameters [3]. A Factor called as 'quality dimensions' is one of the major factors in evaluation of organizational performance accounts for major cumulative variance, this includes such elements as scrap, rework and waste [4]. Among the several possible defects in castings produced by a green sand casting process, some defects are persisting and more challenging to avoid.The occurrences of these defects are seen even though castings are produced for extensive period of time.For

*E-mail address: tomas.ivaniga@tuke.sk

ISSN: $1791-2377$ @ 2019 School of Science, IHU. All rights reserved.

doi:10.25103/jestr.125.15 example the prediction of shrinkage porosity or closed shrinkage in the complex geometrical components such as the cylinder head is not straightforward in general. Porosity is the most persistent and common complaint of casting users. The final reliability of a casting and mechanical properties of the casting is greatly influenced by the presence of defects such as porosity, blowholes, sand inclusions and low hardness. These defects not only cause the loss in productivity and increased production cost, but also affect the performance of these components [5].

In order to minimize persisting defects, Taguchi approach to parameter design is applied in this study to obtain the optimal settings of process parameters. The proposed methodology suggests a selection of potential process parameters in the first step. The next step is experimentation for the evaluation of the individual parameters to optimize the response, quality characteristics. In this study 19 potential parameters are selected at mixed levels and experimentation is performed as per the plan given by chosen Taguchi array, L36 OA $[6,7]$. Results are analyzed using the Taguchi approach to determine optimal levels and ANOVA is used for identification of most influential parameters on the basis of percentage contributions they made in the occurrences of respective persisting defects.

\section{Literature review}

Most of the previous work in the domain of optimization of process parameters of green sand casting process focuses on minimization of casting rejection percentage due to the presence of defects and quality improvement of cast components. A number of researchers work on the optimal 
setting of sand casting process parameters over the past few decades [8]. In work done and published in last ten years, a Taguchi DOE technique and other techniques that are Artificial Neural Networks (ANN) \& Genetic Algorithms (GA) are also used in order to set the optimal level of desired input parameters.In order to investigate the effectiveness of molasses, Mandal and Roy [9] in their work used central composite design (CCD) and Back propagation neural network (BPNN) with different settings of inputs such as molasses, cement and setting time to predict the compressive strength of the sand mix through S. Guharaja et al. [10] minimized the casting defects in SG Iron cast components by using the Taguchi approach for the optimal settings of green sand casting process parameters. In his work total four (4) parameters associated with green sand and mould are selected as input parameters to minimize casting defects. A comparative study was conducted by Karunakar and Datta [11] by using ANN and Genetic Algorithms(GA) to determine the set of desired mould properties and successfully predicted the set of controlling parameters. The results predicted by the GA are more accurate as compared to results obtained by ANN approach. Parappagoudar et al. [12] utilized back-propagation neural network (BP-NN) and genetic-neural network (GA-NN) to model green sand mold system in the forward as well as reverse mapping to predict the responses and to predict the set of input parameters respectively. A. Noorul Haq et al. [13] is employed Taguchi DOE technique for optimization of process parameters in $\mathrm{CO} 2$ sand casting process for minimization of casting defects. In his work total four (4) parameters consider for optimizing properties of $\mathrm{CO} 2$ sand. B. Senthilkumar et al. [14] analyzed identified factors using 'Design of Experiments' approach. Robust design factor values were estimated from the 'signal-to noise' calculations. In his work total three (3) factors related to melting, pouring and methoding (Gating System) are selected for optimization to minimize pull down defect or external shrinkage at the surface. Sushil Kumar et al. [15] in his work proposed Taguchi parameter design approach for optimization of process parameters of green sand casting process to minimize defects of a cast iron differential housing cover. In this work total five (5) parameters are identified, related to molding sand, mold and pouring. Charnnarong et al. [16] in his work optimized sand properties using a mixture of experimental design with the help of Taguchi orthogonal array (OA), RSM, and POE in order to reduce sand related defects on the iron castings. Upadhye et al. [17] in his work applied robust design approach defined by Taguchi to find optimal settings of factors of process and interactions among them with a small number of experiments conducted as per chosen orthogonal array. In his work total eight (8) parameters are selected which are related with green sand, mould \& pouring for optimization to minimize casting defects. Uday A. Dabade et al. [18] effectively used Design of experiment method such as the Taguchi method for deciding the optimum settings of process parameters to have a minimum rejection of castings due to the presence of defects. In his work total four (4) parameters are considered to minimize shrinkage porosity defect in castings. A. Kumaravadivel et al. [19] in his research work investigated the influence of various process parameters on casting defects in the sand-casting operation and optimizing the process parameters to reduce the flywheel defect rejection percentage of the green sand-casting process. In his work Taguchi method of experimental design is applied to analyze the optimum levels of individual process parameters. Ganesh
G. Patil et al. [20] in his work performed as suggested by L18 Taguchi orthogonal array at different combinations of selected process parameters to analyze the contribution of process parameters in occurrences of defects for minimization of rejection in the cast components. In his work total four (4) parameters associated with green sand and mould are identified to minimize casting defects. Manjunath Patel et al. [21] in his work adopted Taguchi parametric design for the squeeze cast technology to yield high density components and superior surface finish by conducting a minimum number of experiments. L9 orthogonal array was adopted to perform the experiments. In this work total three (3) parameters are identified to minimize defects that are; surface roughness and density. Sanjiv Tiwari et al. [22] in his study uses the Taguchi technique of parameter design and determine the optimal settings of process parameters in order to minimize the casting defect for green sand casting process. In this work total five (5) parameters are considered out of which four (4) are related with molding sand and mould while one is related to pouring. A. Johnson et al. [23] To find the optimized levels of various casting parameters in his work to reduce rejection rates in the ductile iron castings, Taguchi L-27 orthogonal array was chosen with 3-level settings of parameters for the analysis. In his work he selected total seven (7) parameters out of which six are related to sand and mould.

\subsection{Research Gap}

After the review of the above significant studies, it was revealed that in most of the work the Taguchi approach proved to be a powerful tool for the optimization of the sand casting process parameters to minimize the casting defects. It is also discovered that, the process parameters associated with principal operations in green sand process are selected for the optimization ranges between 3 to 10 with their values defined at 2 or 3 levels that involved in the process.

While the process parameters affecting the casting defects in the green sand process are generally known, any of the studies published on this domain, hardly, cover the optimization of process parameters associated with all the principal operations involved in the green sand process and recommend the most influential parameters that are responsible for the occurrences of respective persisting defects. In the present work, a total of nineteen green sand casting process parameters are selected that are associated with all the principal operations involved in the process and are alleged to be responsible for the occurrence of the persisting defects. Taguchi L36 OA is used for experimentation. The experimental results and values are converted into the $\mathrm{S} / \mathrm{N}$ ratio to determine the optimal level of the parameters in the Taguchi method and consequently, most influential parameters that are responsible for the occurrences of particular persisting defects are also identified. Study concludes with the confirmation run of experiments to verify the results.

\section{Selection of process parameters}

In foundry industry, the inspection activity in the initial stage is performed just after shakeout operation, i.e. operation performed to separate out the casting from the mould after solidification of metal poured. In latter stage inspection activity is performed after shot blasting i.e. operation performed to clean the moulding sand stick on the casting surface by blasting steel shots on the surface. Daily 
inspection reports indicate the component wise numbers inspected in these two stages and quantity of castings found acceptable and rejected. Details regarding component-wise numbers rejected along with the type of defect responsible for rejection of the component is also mentioned in this report. Quantity rejected at this level is also called as foundry rejection. At the next level, inspection activity is performed after machining operations performed on the castings and quantity rejected is also called machining or customer rejection if machining operation is performed at customer end .In this stage, rejection of castings mainly due to the presence of defects like shrinkage porosity and inclusions which usually lie in the cross section or subsurface area and visible only after machining. During machining castings are also rejected due to occurrence of hard spots (localized excess hardness), difficult to machine .In some cases, defects like shrinkage porosity are also exposed during leak test or pressure test that is mandatory for vital cast components like cylinder heads and performed after machining operations. Apart from this cast components are also rejected because of failure in the field, and called as field rejection. In this study a large amount of data regarding casting rejection is analyzed to expose persisting defects in the foundry industry [6]. From the analysis of large amount of data available with industry regarding casting rejection at foundry levels as well as rejection at customer-end, it is revealed that shrinkage porosity, blowholes, sand inclusion and low hardness are persisting defects and mostly found in complex geometrical components such as cylinder heads. Further analysis of these defects revealed that, defects are associated with different principal operations required to perform in producing cast components with green sand process [7, 12].So, first of all, the possible casting defects in green sand casting process are classified into different groups on the basis of their association with individual process or operation as shown in Table 1. Now, after grouping the defects as shown in Table 1 given below, to analyze the effect of potential process parameters on persisting casting defects, the parameters are selected on the basis of the literature reviewed and opinion by experienced foundry personnel. Table 2 given below indicates the parameters associated with persisting defects.

Table 1. Casting Defects associated with the different operation's in the green sand process

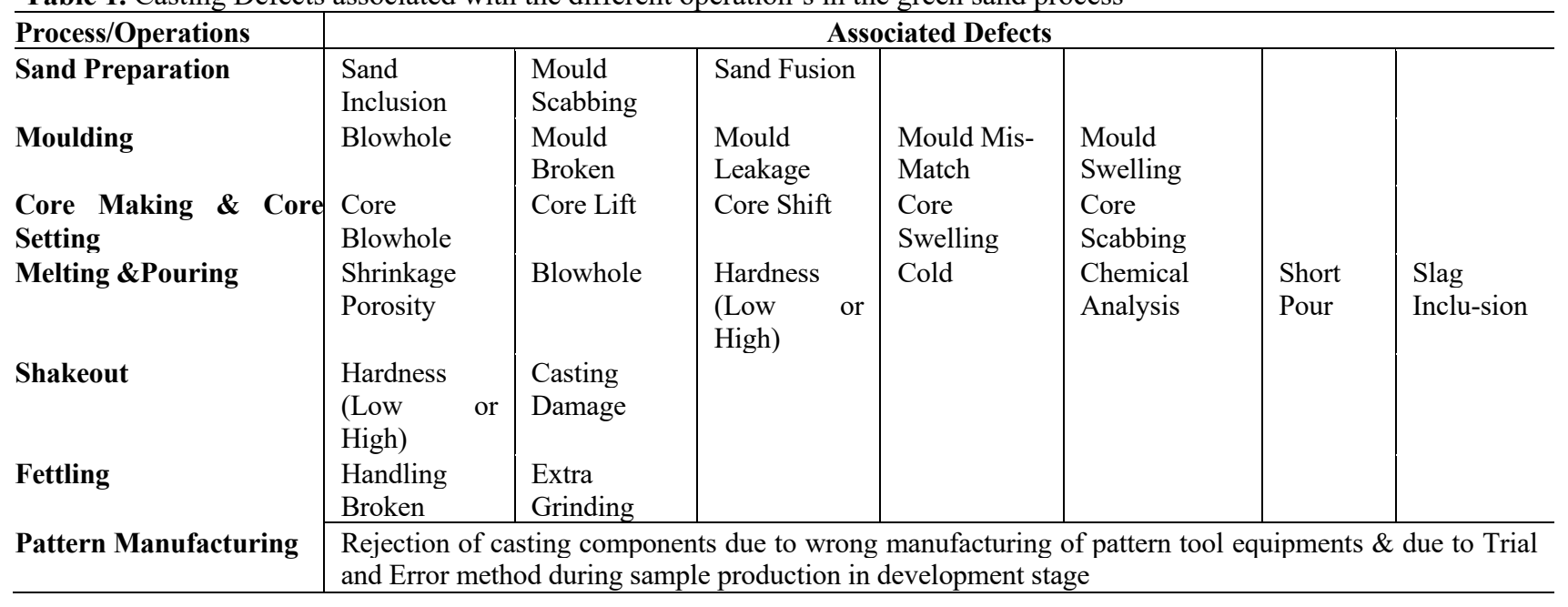

Table 2. Selected process parameters and corresponding levels

\begin{tabular}{|c|c|c|c|c|c|c|}
\hline Sr. No. & Process Parameter & $\begin{array}{c}\text { Parameter } \\
\text { Designation }\end{array}$ & Range & Level 1 & Level 2 & Level 3 \\
\hline 1 & Carbon Equivalent Value (nu) & $\mathrm{A}$ & $3.9-4.0$ & 3.9 & 4.0 & --- \\
\hline 2 & Carbon Content $(\%)$ & $\mathrm{B}$ & $3.3-3.4$ & 3.3 & 3.4 & --- \\
\hline 3 & Pouring Temperature $\left({ }^{0} \mathrm{C}\right)$ & $\mathrm{C}$ & $1400-1410$ & 1400 & 1410 & --- \\
\hline 4 & Inoculants Addition (\%) & $\mathrm{D}$ & $0.15-0.20$ & 0.15 & 0.20 & --- \\
\hline 5 & Steel Scrap Addition (\%) & E & $30-40$ & 30 & 40 & --- \\
\hline 6 & Phosphorus Content (\%) & $\mathrm{F}$ & $0.08-0.09$ & 0.08 & 0.09 & --- \\
\hline 7 & Binder Qty in core sand $(\%)$ & $\mathrm{G}$ & $1.8-2.2$ & 1.8 & 2.2 & --- \\
\hline 8 & Moisture Content (\%) & $\mathrm{H}$ & $4-4.8$ & 4 & 4.4 & 4.8 \\
\hline 9 & Permeability (nu) & $\mathrm{J}$ & $130-150$ & 130 & 140 & 150 \\
\hline 10 & Sulphur Content (\%) & $\mathrm{K}$ & $0.07-0.09$ & 0.07 & 0.08 & 0.09 \\
\hline 11 & Green Compression Strength $\left(\mathrm{Kg} / \mathrm{cm}^{2}\right)$ & $\mathrm{L}$ & $1.4-1.8$ & 1.4 & 1.6 & 1.8 \\
\hline 12 & Compatibility $(\%)$ & M & $40-46$ & 40 & 43 & 46 \\
\hline 13 & Return Sand Temperature $\left({ }^{0} \mathrm{C}\right)$ & $\mathrm{N}$ & $30-50$ & 30 & 40 & 50 \\
\hline 14 & Squeezing Pressure (bar) & $\mathrm{O}$ & $90-110$ & 90 & 100 & 110 \\
\hline 15 & Mould Hardness Horizontal (nu) & $\mathrm{P}$ & $80-90$ & 80 & 85 & 90 \\
\hline 16 & Mould Hardness Vertical (nu) & Q & $70-80$ & 70 & 75 & 80 \\
\hline 17 & Silica content in Sand $(\%)$ & $\mathrm{R}$ & $95-98$ & 95 & 96.5 & 98 \\
\hline 18 & Knockout or Cooling Time (min) & $\mathrm{S}$ & $30-40$ & 30 & 35 & 40 \\
\hline 19 & Manganese Content $(\%)$ & $\mathrm{T}$ & $0.6-0.8$ & 0.6 & 0.7 & 0.8 \\
\hline
\end{tabular}

\section{Selection of orthogonal array}

Total 19 potential parameters are identified in the first stage of study as they are expected to be responsible for the occurrences of persisting defects. Now, as proposed in the 
Taguchi approach to parameter design, an experiment can be performed using suitable Taguchi Orthogonal Array. While selecting a particular orthogonal array for conducting the experiments, two points must be considered that are; the number of parameters \& the number of levels for the parameters of interest. As mentioned in Table 2 total parameters involved in this study are 19 and therefore it was decided to go with L36 array, one of the suitable arrays for 19 parameters with mixed levels [24]. As mentioned in Table 3 , seven parameters are considered at two levels and remaining twelve parameters are considered at three levels for experimentation

The Taguchi method was applied to the experimental data using statistical software "MINITAB 14". The template for experimental plan for 19 parameters with mixed levels using the Taguchi L36 orthogonal array is given below in Table 3, where $-1,0 \&+1$ represents level 1 , level 2 and level 3 of corresponding process parameters. The actual design of experimentation can be obtained by putting values of process parameters at corresponding level as shown in Table 4.

Table 3 Template, Taguchi L36 Array

\begin{tabular}{|c|c|c|c|c|c|c|c|c|c|c|c|c|c|c|c|c|c|c|c|}
\hline $\begin{array}{l}\text { Sr. } \\
\text { No. }\end{array}$ & $\mathbf{A}$ & B & $\mathbf{C}$ & D & $\mathbf{E}$ & $\mathbf{F}$ & $\mathbf{G}$ & $\mathbf{H}$ & $\mathbf{J}$ & $\mathbf{K}$ & $\mathbf{L}$ & $\mathbf{M}$ & $\mathbf{N}$ & $\mathbf{O}$ & $\mathbf{P}$ & $\mathbf{Q}$ & $\mathbf{R}$ & $\mathbf{S}$ & $\mathbf{T}$ \\
\hline 1 & -1 & -1 & -1 & -1 & -1 & -1 & -1 & -1 & -1 & -1 & -1 & -1 & -1 & -1 & -1 & -1 & -1 & -1 & -1 \\
\hline 2 & -1 & -1 & -1 & -1 & -1 & -1 & -1 & 0 & 0 & 0 & 0 & 0 & 0 & 0 & 0 & 0 & 0 & 0 & 0 \\
\hline 3 & -1 & -1 & -1 & -1 & -1 & -1 & -1 & 1 & 1 & 1 & 1 & 1 & 1 & 1 & 1 & 1 & 1 & 1 & 1 \\
\hline 4 & -1 & -1 & -1 & -1 & -1 & 0 & 0 & -1 & -1 & -1 & -1 & 0 & 0 & 0 & 0 & 1 & 1 & 1 & 1 \\
\hline 5 & -1 & -1 & -1 & -1 & -1 & 0 & 0 & 0 & 0 & 0 & 0 & 1 & 1 & 1 & 1 & -1 & -1 & -1 & -1 \\
\hline 6 & -1 & -1 & -1 & -1 & -1 & 0 & 0 & 1 & 1 & 1 & 1 & -1 & -1 & -1 & -1 & 0 & 0 & 0 & 0 \\
\hline 7 & -1 & -1 & 0 & 0 & 0 & -1 & -1 & -1 & -1 & 0 & 1 & -1 & 0 & 1 & 1 & -1 & 0 & 0 & 1 \\
\hline 8 & -1 & -1 & 0 & 0 & 0 & -1 & -1 & 0 & 0 & 1 & -1 & 0 & 1 & -1 & -1 & 0 & 1 & 1 & -1 \\
\hline 9 & -1 & -1 & 0 & 0 & 0 & -1 & -1 & 1 & 1 & -1 & 0 & 1 & -1 & 0 & 0 & 1 & -1 & -1 & 0 \\
\hline 10 & -1 & 0 & -1 & 0 & 0 & -1 & 0 & -1 & -1 & 1 & 0 & -1 & 1 & 0 & 1 & 0 & -1 & 1 & 0 \\
\hline 11 & -1 & 0 & -1 & 0 & 0 & -1 & 0 & 0 & 0 & -1 & 1 & 0 & -1 & 1 & -1 & 1 & 0 & -1 & 1 \\
\hline 12 & -1 & 0 & -1 & 0 & 0 & -1 & 0 & 1 & 1 & 0 & -1 & 1 & 0 & -1 & 0 & -1 & 1 & 0 & -1 \\
\hline 13 & -1 & 0 & 0 & -1 & 0 & 0 & -1 & -1 & 0 & 1 & -1 & 1 & 0 & -1 & 1 & 1 & 0 & -1 & 0 \\
\hline 14 & -1 & 0 & 0 & -1 & 0 & 0 & -1 & 0 & 1 & -1 & 0 & -1 & 1 & 0 & -1 & -1 & 1 & 0 & 1 \\
\hline 15 & -1 & 0 & 0 & -1 & 0 & 0 & -1 & 1 & -1 & 0 & 1 & 0 & -1 & 1 & 0 & 0 & -1 & 1 & -1 \\
\hline 16 & -1 & 0 & 0 & 0 & -1 & 0 & 0 & -1 & 0 & 1 & 0 & -1 & -1 & 1 & 0 & 1 & 1 & 0 & -1 \\
\hline 17 & -1 & 0 & 0 & 0 & -1 & 0 & 0 & 0 & 1 & -1 & 1 & 0 & 0 & -1 & 1 & -1 & -1 & 1 & 0 \\
\hline 18 & -1 & 0 & 0 & 0 & -1 & 0 & 0 & 1 & -1 & 0 & -1 & 1 & 1 & 0 & -1 & 0 & 0 & -1 & 1 \\
\hline 19 & 0 & -1 & 0 & 0 & -1 & -1 & 0 & -1 & 0 & -1 & 1 & 1 & 1 & -1 & 0 & 0 & -1 & 0 & 1 \\
\hline 20 & 0 & -1 & 0 & 0 & -1 & -1 & 0 & 0 & 1 & 0 & -1 & -1 & -1 & 0 & 1 & 1 & 0 & 1 & -1 \\
\hline 21 & 0 & -1 & 0 & 0 & -1 & -1 & 0 & 1 & -1 & 1 & 0 & 0 & 0 & 1 & -1 & -1 & 1 & -1 & 0 \\
\hline 22 & 0 & -1 & 0 & -1 & 0 & 0 & 0 & -1 & 0 & 0 & 1 & 1 & -1 & 0 & -1 & -1 & 1 & 1 & 0 \\
\hline 23 & 0 & -1 & 0 & -1 & 0 & 0 & 0 & 0 & 1 & 1 & -1 & -1 & 0 & 1 & 0 & 0 & -1 & -1 & 1 \\
\hline 24 & 0 & -1 & 0 & -1 & 0 & 0 & 0 & 1 & -1 & -1 & 0 & 0 & 1 & -1 & 1 & 1 & 0 & 0 & -1 \\
\hline 25 & 0 & -1 & -1 & 0 & 0 & 0 & -1 & -1 & 1 & 0 & -1 & 0 & 1 & 1 & -1 & 1 & -1 & 0 & 0 \\
\hline 26 & 0 & -1 & -1 & 0 & 0 & 0 & -1 & 0 & -1 & 1 & 0 & 1 & -1 & -1 & 0 & -1 & 0 & 1 & 1 \\
\hline 27 & 0 & -1 & -1 & 0 & 0 & 0 & -1 & 1 & 0 & -1 & 1 & -1 & 0 & 0 & 1 & 0 & 1 & -1 & -1 \\
\hline 28 & 0 & 0 & 0 & -1 & -1 & -1 & -1 & -1 & 1 & 0 & 0 & 0 & -1 & -1 & 1 & 0 & 1 & -1 & 1 \\
\hline 29 & 0 & 0 & 0 & -1 & -1 & -1 & -1 & 0 & -1 & 1 & 1 & 1 & 0 & 0 & -1 & 1 & -1 & 0 & -1 \\
\hline 30 & 0 & 0 & 0 & -1 & -1 & -1 & -1 & 1 & 0 & -1 & -1 & -1 & 1 & 1 & 0 & -1 & 0 & 1 & 0 \\
\hline 31 & 0 & 0 & -1 & 0 & -1 & 0 & -1 & -1 & 1 & 1 & 1 & 0 & 1 & 0 & 0 & -1 & 0 & -1 & -1 \\
\hline 32 & 0 & 0 & -1 & 0 & -1 & 0 & -1 & 0 & -1 & -1 & -1 & 1 & -1 & 1 & 1 & 0 & 1 & 0 & 0 \\
\hline 33 & 0 & 0 & -1 & 0 & -1 & 0 & -1 & 1 & 0 & 0 & 0 & -1 & 0 & -1 & -1 & 1 & -1 & 1 & 1 \\
\hline 34 & 0 & 0 & -1 & -1 & 0 & -1 & 0 & -1 & 1 & -1 & 0 & 1 & 0 & 1 & -1 & 0 & 0 & 1 & -1 \\
\hline 35 & 0 & 0 & -1 & -1 & 0 & -1 & 0 & 0 & -1 & 0 & 1 & -1 & 1 & -1 & 0 & 1 & 1 & -1 & 0 \\
\hline 36 & 0 & 0 & -1 & -1 & 0 & -1 & 0 & 1 & 0 & 1 & -1 & 0 & -1 & 0 & 1 & -1 & -1 & 0 & 1 \\
\hline
\end{tabular}

Source: Madhav S. Phadke (2009), Quality Engineering Using Robust Design, Pearson Publication, India

\section{Experimentation}

Actual experimentation can be performed after the parameters assigned to a particular column of the selected orthogonal array, as shown in Table 4, [7, 24].The casting of Cylinder Head (3 Bore) was made against the experimental plan given in Table No.4. As per chosen array L36, 36 experiments are conducted and experimental outcomes are recorded, in the present work outcomes are a percentage of castings rejected due to presence of respective persisting defects. Casting rejection percentage due to the presence of defects for each experimentation was recorded and then by using the given formula, rejection percentage due to presence of respective persisting defects were calculated for each trial condition which is a ratio of the number of castings rejected due to presence of respective Persisting defects to the total number of castings rejected.

Percentage $=$ No. of castings rejected due to Persistingof respective persisting defects $* 100$ 
Table 4 Actual Experimentation Plan

\begin{tabular}{|c|c|c|c|c|c|c|c|c|c|c|c|c|c|c|c|c|c|c|c|}
\hline $\begin{array}{l}\text { Sr. } \\
\text { No. }\end{array}$ & $\mathbf{A}$ & B & $\mathrm{C}$ & D & $\mathbf{E}$ & $\mathbf{F}$ & G & H & $\mathbf{J}$ & $\mathbf{K}$ & $\mathbf{L}$ & $\mathbf{M}$ & $\mathbf{N}$ & $\mathbf{O}$ & $\mathbf{P}$ & $\mathbf{Q}$ & $\mathbf{R}$ & $\mathbf{S}$ & $\mathbf{T}$ \\
\hline 1 & 3.9 & 3.3 & 1400 & 0.15 & 30 & 0.08 & 1.8 & 4.00 & 130 & 0.07 & 1.4 & 40.0 & 30 & 90 & 80 & 70 & 95.0 & 30 & 0.6 \\
\hline 2 & 3.9 & 3.3 & 1400 & 0.15 & 30 & 0.08 & 1.8 & 4.40 & 140 & 0.08 & 1.6 & 43.0 & 40 & 100 & 85 & 75 & 96.5 & 35 & 0.7 \\
\hline 3 & 3.9 & 3.3 & 1400 & 0.15 & 30 & 0.08 & 1.8 & 4.80 & 150 & 0.09 & 1.8 & 46.0 & 50 & 110 & 90 & 80 & 98.0 & 40 & 0.8 \\
\hline 4 & 3.9 & 3.3 & 1400 & 0.15 & 30 & 0.09 & 2.2 & 4.00 & 130 & 0.07 & 1.4 & 43.0 & 40 & 100 & 85 & 80 & 98.0 & 40 & 0.8 \\
\hline 5 & 3.9 & 3.3 & 1400 & 0.15 & 30 & 0.09 & 2.2 & 4.40 & 140 & 0.08 & 1.6 & 46.0 & 50 & 110 & 90 & 70 & 95.0 & 30 & 0.6 \\
\hline 6 & 3.9 & 3.3 & 1400 & 0.15 & 30 & 0.09 & 2.2 & 4.80 & 150 & 0.09 & 1.8 & 40.0 & 30 & 90 & 80 & 75 & 96.5 & 35 & 0.7 \\
\hline 7 & 3.9 & 3.3 & 1410 & 0.20 & 40 & 0.08 & 1.8 & 4.00 & 130 & 0.08 & 1.8 & 40.0 & 40 & 110 & 90 & 70 & 96.5 & 35 & 0.8 \\
\hline 8 & 3.9 & 3.3 & 1410 & 0.20 & 40 & 0.08 & 1.8 & 4.40 & 140 & 0.09 & 1.4 & 43.0 & 50 & 90 & 80 & 75 & 98.0 & 40 & 0.6 \\
\hline 9 & 3.9 & 3.3 & 1410 & 0.20 & 40 & 0.08 & 1.8 & 4.80 & 150 & 0.07 & 1.6 & 46.0 & 30 & 100 & 85 & 80 & 95.0 & 30 & 0.7 \\
\hline 10 & 3.9 & 3.4 & 1400 & 0.20 & 40 & 0.08 & 2.2 & 4.00 & 130 & 0.09 & 1.6 & 40.0 & 50 & 100 & 90 & 75 & 95.0 & 40 & 0.7 \\
\hline 11 & 3.9 & 3.4 & 1400 & 0.20 & 40 & 0.08 & 2.2 & 4.40 & 140 & 0.07 & 1.8 & 43.0 & 30 & 110 & 80 & 80 & 96.5 & 30 & 0.8 \\
\hline 12 & 3.9 & 3.4 & 1400 & 0.20 & 40 & 0.08 & 2.2 & 4.80 & 150 & 0.08 & 1.4 & 46.0 & 40 & 90 & 85 & 70 & 98.0 & 35 & 0.6 \\
\hline 13 & 3.9 & 3.4 & 1410 & 0.15 & 40 & 0.09 & 1.8 & 4.00 & 140 & 0.09 & 1.4 & 46.0 & 40 & 90 & 90 & 80 & 96.5 & 30 & 0.7 \\
\hline 14 & 3.9 & 3.4 & 1410 & 0.15 & 40 & 0.09 & 1.8 & 4.40 & 150 & 0.07 & 1.6 & 40.0 & 50 & 100 & 80 & 70 & 98.0 & 35 & 0.8 \\
\hline 15 & 3.9 & 3.4 & 1410 & 0.15 & 40 & 0.09 & 1.8 & 4.80 & 130 & 0.08 & 1.8 & 43.0 & 30 & 110 & 85 & 75 & 95.0 & 40 & 0.6 \\
\hline 16 & 3.9 & 3.4 & 1410 & 0.20 & 30 & 0.09 & 2.2 & 4.00 & 140 & 0.09 & 1.6 & 40.0 & 30 & 110 & 85 & 80 & 98.0 & 35 & 0.6 \\
\hline 17 & 3.9 & 3.4 & 1410 & 0.20 & 30 & 0.09 & 2.2 & 4.40 & 150 & 0.07 & 1.8 & 43.0 & 40 & 90 & 90 & 70 & 95.0 & 40 & 0.7 \\
\hline 18 & 3.9 & 3.4 & 1410 & 0.20 & 30 & 0.09 & 2.2 & 4.80 & 130 & 0.08 & 1.4 & 46.0 & 50 & 100 & 80 & 75 & 96.5 & 30 & 0.8 \\
\hline 19 & 4.0 & 3.3 & 1410 & 0.20 & 30 & 0.08 & 2.2 & 4.00 & 140 & 0.07 & 1.8 & 46.0 & 50 & 90 & 85 & 75 & 95.0 & 35 & 0.8 \\
\hline 20 & 4.0 & 3.3 & 1410 & 0.20 & 30 & 0.08 & 2.2 & 4.40 & 150 & 0.08 & 1.4 & 40.0 & 30 & 100 & 90 & 80 & 96.5 & 40 & 0.6 \\
\hline 21 & 4.0 & 3.3 & 1410 & 0.20 & 30 & 0.08 & 2.2 & 4.80 & 130 & 0.09 & 1.6 & 43.0 & 40 & 110 & 80 & 70 & 98.0 & 30 & 0.7 \\
\hline 22 & 4.0 & 3.3 & 1410 & 0.15 & 40 & 0.09 & 2.2 & 4.00 & 140 & 0.08 & 1.8 & 46.0 & 30 & 100 & 80 & 70 & 98.0 & 40 & 0.7 \\
\hline 23 & 4.0 & 3.3 & 1410 & 0.15 & 40 & 0.09 & 2.2 & 4.40 & 150 & 0.09 & 1.4 & 40.0 & 40 & 110 & 85 & 75 & 95.0 & 30 & 0.8 \\
\hline 24 & 4.0 & 3.3 & 1410 & 0.15 & 40 & 0.09 & 2.2 & 4.80 & 130 & 0.07 & 1.6 & 43.0 & 50 & 90 & 90 & 80 & 96.5 & 35 & 0.6 \\
\hline 25 & 4.0 & 3.3 & 1400 & 0.20 & 40 & 0.09 & 1.8 & 4.00 & 150 & 0.08 & 1.4 & 43.0 & 50 & 110 & 80 & 80 & 95.0 & 35 & 0.7 \\
\hline 26 & 4.0 & 3.3 & 1400 & 0.20 & 40 & 0.09 & 1.8 & 4.40 & 130 & 0.09 & 1.6 & 46.0 & 30 & 90 & 85 & 70 & 96.5 & 40 & 0.8 \\
\hline 27 & 4.0 & 3.3 & 1400 & 0.20 & 40 & 0.09 & 1.8 & 4.80 & 140 & 0.07 & 1.8 & 40.0 & 40 & 100 & 90 & 75 & 98.0 & 30 & 0.6 \\
\hline 28 & 4.0 & 3.4 & 1410 & 0.15 & 30 & 0.08 & 1.8 & 4.00 & 150 & 0.08 & 1.6 & 43.0 & 30 & 90 & 90 & 75 & 98.0 & 30 & 0.8 \\
\hline 29 & 4.0 & 3.4 & 1410 & 0.15 & 30 & 0.08 & 1.8 & 4.40 & 130 & 0.09 & 1.8 & 46.0 & 40 & 100 & 80 & 80 & 95.0 & 35 & 0.6 \\
\hline 30 & 4.0 & 3.4 & 1410 & 0.15 & 30 & 0.08 & 1.8 & 4.80 & 140 & 0.07 & 1.4 & 40.0 & 50 & 110 & 85 & 70 & 96.5 & 40 & 0.7 \\
\hline 31 & 4.0 & 3.4 & 1400 & 0.20 & 30 & 0.09 & 1.8 & 4.00 & 150 & 0.09 & 1.8 & 43.0 & 50 & 100 & 85 & 70 & 96.5 & 30 & 0.6 \\
\hline 32 & 4.0 & 3.4 & 1400 & 0.20 & 30 & 0.09 & 1.8 & 4.40 & 130 & 0.07 & 1.4 & 46.0 & 30 & 110 & 90 & 75 & 98.0 & 35 & 0.7 \\
\hline 33 & 4.0 & 3.4 & 1400 & 0.20 & 30 & 0.09 & 1.8 & 4.80 & 140 & 0.08 & 1.6 & 40.0 & 40 & 90 & 80 & 80 & 95.0 & 40 & 0.8 \\
\hline 34 & 4.0 & 3.4 & 1400 & 0.15 & 40 & 0.08 & 2.2 & 4.00 & 150 & 0.07 & 1.6 & 46.0 & 40 & 110 & 80 & 75 & 96.5 & 40 & 0.6 \\
\hline 35 & 4.0 & 3.4 & 1400 & 0.15 & 40 & 0.08 & 2.2 & 4.40 & 130 & 0.08 & 1.8 & 40.0 & 50 & 90 & 85 & 80 & 98.0 & 30 & 0.7 \\
\hline 36 & 4.0 & 3.4 & 1400 & 0.15 & 40 & 0.08 & 2.2 & 4.80 & 140 & 0.09 & 1.4 & 43.0 & 30 & 100 & 90 & 70 & 95.0 & 35 & 0.8 \\
\hline
\end{tabular}

6 Taguchi analysis of experimentation output

Table 5. Casting defects and corresponding $\mathrm{S} / \mathrm{N}$ ratio

\begin{tabular}{|c|c|c|c|c|c|c|c|c|}
\hline \multirow{2}{*}{$\begin{array}{l}\text { Exp. } \\
\text { No. }\end{array}$} & \multicolumn{8}{|c|}{ Avg. Value in \% for Persisting Defects \& Corresponding S/N Ratio } \\
\hline & $\begin{array}{l}\text { ShrinkagePorosity } \\
\text { (Y1) }\end{array}$ & $\begin{array}{l}\text { S/N } \\
\text { Ratio }\end{array}$ & $\begin{array}{l}\text { Blowhole } \\
\text { (Y2) } \\
\text { (Y2) }\end{array}$ & $\begin{array}{l}\text { S/N } \\
\text { Ratio }\end{array}$ & $\begin{array}{l}\text { Sand } \\
\text { Inclusion } \\
\text { (Y3) }\end{array}$ & $\begin{array}{l}\text { S/N } \\
\text { Ratio }\end{array}$ & $\begin{array}{l}\text { Low } \\
\text { Hardness } \\
\text { (Y4) }\end{array}$ & $\begin{array}{l}\text { S/N } \\
\text { Ratio }\end{array}$ \\
\hline 1 & 3.8 & -11.5957 & 0.7 & 3.0980 & 1.06 & -0.5061 & 0.45 & 6.9357 \\
\hline 2 & 3.33 & -10.4489 & 1 & 0.0000 & 0.89 & 1.0122 & 0.28 & 11.0568 \\
\hline 3 & 3 & -9.5424 & 1.04 & -0.3407 & 0.89 & 1.0122 & 0.18 & 14.8945 \\
\hline 4 & 3.67 & -11.2933 & 1.6 & -4.0824 & 1.12 & -0.9844 & 0.22 & 13.1515 \\
\hline 5 & 3.8 & -11.5957 & 1.17 & -1.3637 & 0.9 & 0.9151 & 0.33 & 9.6297 \\
\hline 6 & 3.56 & -11.0290 & 1.07 & -0.5877 & 0.86 & 1.3100 & 0.26 & 11.7005 \\
\hline 7 & 3.32 & -10.4228 & 1.05 & -0.4238 & 0.98 & 0.1755 & 0.24 & 12.3958 \\
\hline 8 & 3.95 & -11.9319 & 1.08 & -0.6685 & 1.3 & -2.2789 & 0.46 & 6.7448 \\
\hline 9 & 4.22 & -12.5062 & 0.98 & 0.1755 & 1.08 & -0.6685 & 0.29 & 10.7520 \\
\hline 10 & 3.82 & -11.6413 & 1.3 & -2.2789 & 1.2 & -1.5836 & 0.27 & 11.3727 \\
\hline 11 & 3.96 & -11.9539 & 1.26 & -2.0074 & 1.06 & -0.5061 & 0.2 & 13.9794 \\
\hline 12 & 4.02 & -12.0845 & 0.98 & 0.1755 & 1.34 & -2.5421 & 0.4 & 7.9588 \\
\hline 13 & 4.5 & -13.0643 & 0.88 & 1.1103 & 1.28 & -2.1442 & 0.3 & 10.4576 \\
\hline 14 & 3.6 & -11.1261 & 1.09 & -0.7485 & 1.09 & -0.7485 & 0.27 & 11.3727 \\
\hline 15 & 4.5 & -13.0643 & 1.05 & -0.4238 & 1.1 & -0.8279 & 0.33 & 9.6297 \\
\hline 16 & 4.21 & -12.4856 & 1.06 & -0.5061 & 0.9 & 0.9151 & 0.36 & 8.8739 \\
\hline 17 & 3.7 & -11.3640 & 1.05 & -0.4238 & 0.98 & 0.1755 & 0.28 & 11.0568 \\
\hline 18 & 3.94 & -11.9099 & 1.74 & -4.8110 & 1.3 & -2.2789 & 0.24 & 12.3958 \\
\hline 19 & 2.68 & -8.5627 & 1.32 & -2.4115 & 1.1 & -0.8279 & 0.25 & 12.0412 \\
\hline 20 & 2.87 & -9.1576 & 1.22 & -1.7272 & 1.11 & -0.9065 & 0.4 & 7.9588 \\
\hline
\end{tabular}




\begin{tabular}{l|l|l|l|l|l|l|l|l}
\hline 21 & 3 & -9.5424 & 1.55 & -3.8066 & 0.89 & 1.0122 & 0.33 & 9.6297 \\
22 & 3.24 & -10.2109 & 1.33 & -2.4770 & 0.9 & 0.9151 & 0.3 & 10.4576 \\
23 & 3.65 & -11.2459 & 1.39 & -2.8603 & 1.1 & -0.8279 & 0.25 & 12.0412 \\
24 & 4.02 & -12.0845 & 1.67 & -4.4543 & 1.3 & -2.2789 & 0.32 & 9.8970 \\
25 & 3.66 & -11.2696 & 0.84 & 1.5144 & 1.28 & -2.1442 & 0.36 & 8.8739 \\
26 & 3.56 & -11.0290 & 0.93 & 0.6303 & 1.1 & -0.8279 & 0.26 & 11.7005 \\
27 & 3.3 & -10.3703 & 0.74 & 2.6154 & 0.98 & 0.1755 & 0.3 & 10.4576 \\
28 & 2.66 & -8.4976 & 0.76 & 2.3837 & 1 & 0.0000 & 0.22 & 13.1515 \\
29 & 3.01 & -9.5713 & 1.1 & -0.8279 & 0.87 & 1.2096 & 0.33 & 9.6297 \\
30 & 2.4 & -7.6042 & 1.3 & -2.2789 & 1.22 & -1.7272 & 0.34 & 9.3704 \\
31 & 2.64 & -8.4321 & 0.52 & 5.6799 & 0.85 & 1.4116 & 0.38 & 8.4043 \\
32 & 3.01 & -9.5713 & 0.87 & 1.2096 & 0.89 & 1.0122 & 0.29 & 10.7520 \\
33 & 2.88 & -9.1878 & 1.16 & -1.2892 & 1.18 & -1.4376 & 0.24 & 12.3958 \\
34 & 2.9 & -9.2480 & 1.24 & -1.8684 & 1.2 & -1.5836 & 0.35 & 9.1186 \\
35 & 3.54 & -10.9801 & 1.6 & -4.0824 & 1.08 & -0.6685 & 0.2 & 13.9794 \\
36 & 3.3 & -10.3703 & 1.46 & -3.2871 & 0.98 & 0.1755 & 0.24 & 12.3958 \\
\hline
\end{tabular}

The Taguchi method uses the signal-to-noise $\mathrm{S} / \mathrm{N}$ ratio instead of the response value obtained as a result of in the optimum setting analysis. The $\mathrm{S} / \mathrm{N}$ ratio replicates both the average and the variation of the response or quality characteristics [7, 24-25]. The percentages of castings rejected due to presence of respective persisting defects that occur in each trial condition were calculated by using equation 1 given above. The casting defects are the "loweris -the better" type of quality characteristics. $\mathrm{S} / \mathrm{N}$ ratios for this condition are calculated by the equation No. 2 given below. $\mathrm{S} / \mathrm{N}$ ratios are calculated for each of the 36 trials and Corresponding $\mathrm{S} / \mathrm{N}$ ratios are given above in Table 5 .

$$
S / N L B \text { ratio }=-10 \log 1 / n[\Sigma y i 2](2)
$$

Where, ' $n$ ' is trial number of observations and ' $y_{i}$ ' is the response or observed data in ' $i$ 'th is the experiment number. After calculating the $\mathrm{S} / \mathrm{N}$ ratios, the average $\mathrm{S} / \mathrm{N}$ value is calculated for each factor at different levels [25-26]. The mean response refers to the average value of the performance characteristics for each parameter summarized in Tables 6 to 9. The average values of $\mathrm{S} / \mathrm{N}$ ratios for individual process parameters at different levels are plotted on response graph and shown in Figures 1 to 4 . From

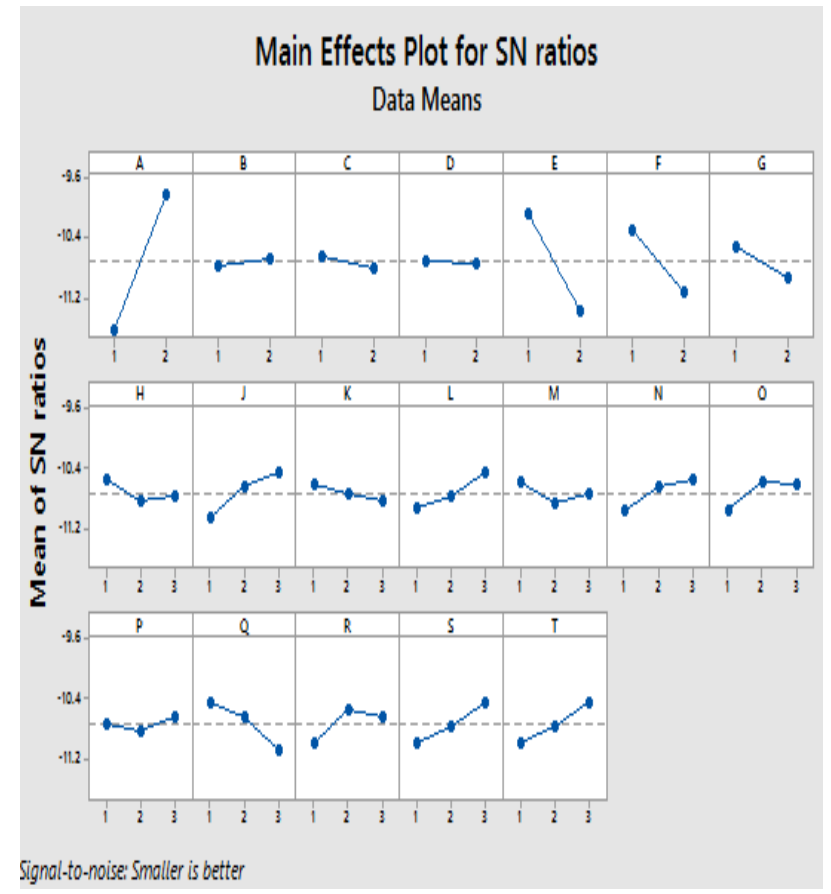

response tables 6 to 9 and Figure 1 to 4 for main effect plots or response graph, it can be concluded that which process parameter level works better to minimize persisting casting defects.

Table 6. S/N ratios at different levels for Shrinkage Porosity (Y1)

\begin{tabular}{l|l|l|l|l|l}
\hline $\begin{array}{l}\text { Process } \\
\text { Variable }\end{array}$ & Level 1 & Level 2 & Level 3 & $\begin{array}{l}\text { Delta } \\
\text { Value }\end{array}$ & Rank \\
\hline A & -11.614 & -9.830 & $\ldots \ldots$ & 1.785 & 1 \\
B & -10.769 & -10.675 & $\ldots \ldots$ & 0.093 & 18 \\
C & -10.647 & -10.797 & $\ldots \ldots$ & 0.151 & 17 \\
D & -10.698 & -10.746 & $\ldots \ldots$. & 0.047 & 19 \\
E & -10.077 & -11.367 & $\ldots \ldots$. & 1.290 & 2 \\
F & -10.315 & -10.315 & $\ldots \ldots$. & 0.815 & 3 \\
G & -10.513 & -10.931 & $\ldots \ldots$ & 0.418 & 10 \\
H & -10.560 & -10.831 & -10.775 & 0.271 & 14 \\
J & -11.059 & -10.649 & -10.459 & 0.600 & 8 \\
K & -10.607 & -10.736 & -10.824 & 0.217 & 15 \\
L & -10.925 & -10.783 & -10.459 & 0.466 & 8 \\
M & -10.571 & -10.854 & -10.741 & 0.284 & 13 \\
N & -10.956 & -10.654 & -10.557 & 0.399 & 11 \\
O & -10.951 & -10.587 & -10.629 & 0.364 & 12 \\
P & -10.715 & -10.811 & -10.640 & 0.171 & 18 \\
Q & -10.448 & -10.627 & -11.091 & 0.643 & 4 \\
R & -10.998 & -10.532 & -10.636 & 0.466 & 9 \\
S & -10.975 & -10.752 & -10.440 & 0.535 & 7 \\
T & -10.968 & -10.769 & -10.428 & 0.540 & 6 \\
\hline
\end{tabular}

Fig. 1. Graph of the main effects of $S / N$ for Shrinkage Porosity(Y1)

Table 7. S/N ratios at different levels for Blowholes (Y2)

\begin{tabular}{l|l|l|l|l|l}
\hline $\begin{array}{l}\text { Process } \\
\text { Factors }\end{array}$ & Level 1 & Level 2 & Level 3 & $\begin{array}{l}\text { Delta } \\
\text { Value }\end{array}$ & Rank \\
\hline A & -0.78371 & -0.96319 & $\ldots \ldots$ & 0.17947 & 16 \\
B & -0.95389 & -0.79301 & $\ldots$. & 0.16088 & 17 \\
C & -0.34803 & -1.39887 & $\ldots \ldots$ & 1.05083 & 7 \\
D & -1.28283 & -0.46407 & $\ldots \ldots$ & 0.81876 & 9 \\
E & -0.67140 & -1.07550 & $\ldots \ldots$ & 0.40410 & 14 \\
F & -1.12091 & -0.62599 & $\ldots \ldots$ & 0.49492 & 13 \\
G & 0.63423 & -2.38113 & $\ldots$ & 3.01536 & 1 \\
H & -0.02180 & -1.07248 & -1.52607 & 1.50427 & 4 \\
J & -1.68775 & -1.04697 & 0.11437 & 1.80212 & 2 \\
K & -0.93139 & -1.04370 & -0.64525 & 0.39845 & 15 \\
L & -1.05062 & -1.09385 & -0.47588 & 0.61797 & 11 \\
M & -0.92246 & -0.79798 & -0.89991 & 0.12447 & 18 \\
N & -0.29326 & -0.97343 & -1.35366 & 1.06041 & 6 \\
O & -0.54328 & -0.98076 & -1.09631 & 0.55303 & 12 \\
P & -1.20664 & -0.83201 & -0.58170 & 0.62495 & 10 \\
Q & -0.43547 & -0.80844 & -1.37644 & 0.94097 & 8 \\
R & -0.86484 & -0.89484 & -0.86067 & 0.03417 & 19 \\
S & -0.32238 & -0.86228 & -1.43569 & 1.11331 & 5 \\
T & -0.02259 & -0.99378 & -1.60398 & 1.58138 & 3 \\
\hline
\end{tabular}




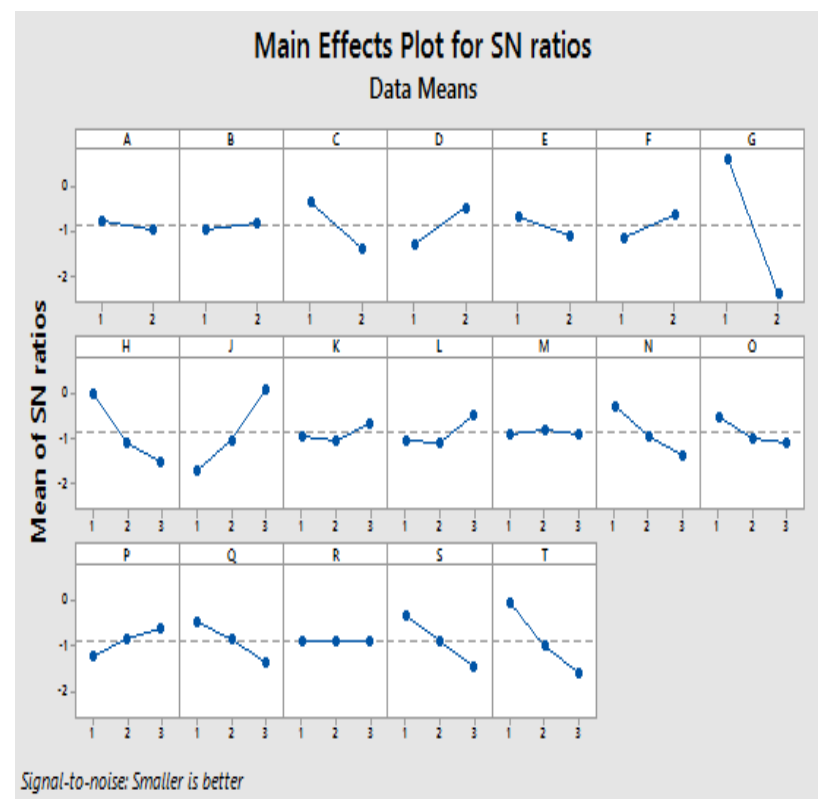

Fig. 2 Graph of the main effects of $S / N$ for Blowhole (Y2)

Table 8. S/N ratios at different levels for Sand Inclusion

\begin{tabular}{l|l|l|l|l|l}
\multicolumn{1}{l}{ (Y3) } \\
\hline $\begin{array}{l}\text { Process } \\
\text { Factors }\end{array}$ & Level 1 & Level 2 & Level 3 & $\begin{array}{l}\text { Delta } \\
\text { Value }\end{array}$ & Rank \\
\hline A & -0.53075 & -0.40657 & $\ldots$. & 0.12417 & 17 \\
B & -0.31795 & -0.61937 & $\ldots .$. & 0.30143 & 11 \\
C & -0.31999 & -0.61733 & $\ldots .$. & 0.29734 & 14 \\
D & -0.31929 & -0.61802 & $\ldots .$. & 0.29873 & 12 \\
E & 0.07319 & -1.01050 & $\ldots .$. & 1.08369 & 2 \\
F & -0.51121 & -0.42611 & $\ldots .$. & 0.08510 & 18 \\
G & -0.40567 & -0.53164 & $\ldots$. & 0.12597 & 16 \\
H & -0.52972 & -0.20329 & -0.67297 & 0.46967 & 9 \\
J & -0.54554 & -0.40111 & -0.45933 & 0.14444 & 15 \\
K & -0.70567 & -0.64897 & -0.05134 & 0.65432 & 5 \\
L & -1.26271 & -0.43949 & 0.29623 & 1.55894 & 1 \\
M & -0.48581 & -0.43611 & -0.48405 & 0.04971 & 19 \\
N & 0.00709 & -0.47994 & -0.93313 & 0.94022 & 3 \\
O & -1.00220 & -0.18923 & -0.21454 & 0.81297 & 4 \\
P & -0.58641 & -0.54692 & -0.27264 & 0.31377 & 10 \\
Q & -0.13094 & -0.55822 & -0.71682 & 0.58588 & 7 \\
R & -0.52899 & -0.69532 & -0.18166 & 0.51366 & 8 \\
S & -0.34047 & -0.22762 & -0.83789 & 0.61027 & 6 \\
T & -0.52474 & -0.29158 & -0.58966 & 0.29808 & 13 \\
\hline \multicolumn{5}{|c|}{}
\end{tabular}

Table 9. S/N ratios at different levels for Low Hardness (Y4)

\begin{tabular}{l|l|l|l|l|l}
\hline $\begin{array}{l}\text { Process } \\
\text { Factors }\end{array}$ & Level 1 & Level 2 & Level 3 & $\begin{array}{l}\text { Delta } \\
\text { Value }\end{array}$ & Rank \\
\hline A & 10.798 & 10.681 & $\ldots$. & 0.117 & 15 \\
B & 10.573 & 10.905 & $\ldots .$. & 0.332 & 12 \\
C & 11.042 & 10.436 & $\ldots \ldots$ & 0.606 & 6 \\
D & 11.048 & 10.430 & $\ldots \ldots$ & 0.618 & 5 \\
E & 10.724 & 10.755 & $\ldots .$. & 0.031 & 18 \\
F & 10.743 & 10.736 & $\ldots \ldots$ & 0.007 & 19 \\
G & 10.499 & 10.980 & $\ldots \ldots$ & 0.481 & 8 \\
H & 10.436 & 10.825 & 10.956 & 0.520 & 7 \\
J & 10.956 & 10.655 & 10.607 & 0.349 & 11 \\
K & 10.740 & 10.824 & 10.654 & 0.170 & 13 \\
L & 9.920 & 10.746 & 11.552 & 1.633 & 2 \\
M & 10.738 & 10.664 & 10.816 & 0.151 & 14 \\
N & 10.691 & 10.779 & 10.748 & 0.089 & 17 \\
O & 10.668 & 10.784 & 10.766 & 0.115 & 16 \\
P & 10.270 & 10.747 & 11.202 & 0.932 & 4 \\
Q & 10.109 & 10.872 & 11.237 & 1.128 & 3 \\
R & 10.563 & 10.703 & 10.952 & 0.389 & 10 \\
S & 10.985 & 10.579 & 10.654 & 0.405 & 9 \\
T & 8.770 & 10.788 & 12.660 & 3.890 & 1 \\
\hline
\end{tabular}

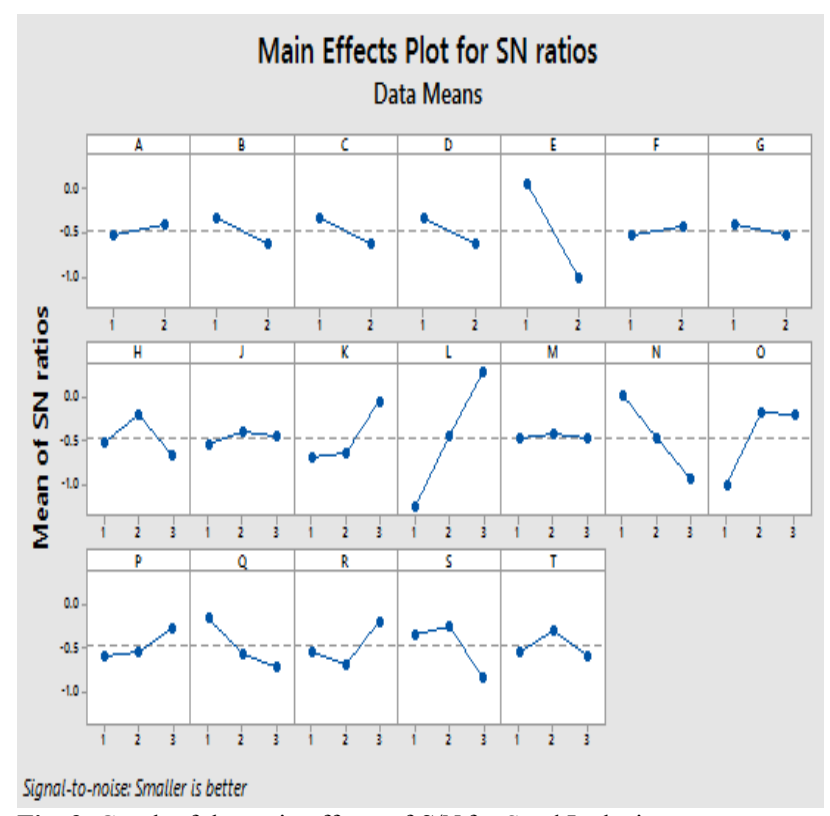

Fig. 3. Graph of the main effects of $S / N$ for Sand Inclusion

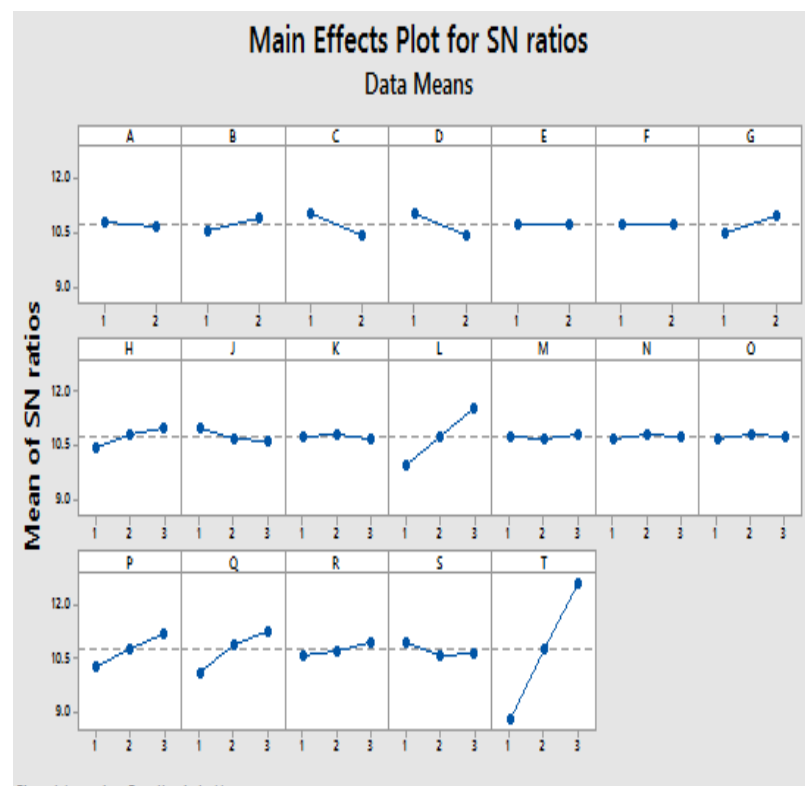

Signal-to-noise: Smaller is better

Fig. 4. Graph of the main effects of $S / N$ for Low Hardness

As mentioned in the response table, the Taguchi method gives the rank of individual parameter and optimal level can also be determined from the response graph for respective quality characteristics. Since the quality characteristics are the lower the better type of characteristics, the maximum $\mathrm{S} / \mathrm{N}$ ratio gives the optimum level for setting of process parameters [30, 31]. But the Taguchi method cannot judge and determine the effect of individual process parameters on the entire process. Therefore, analysis of variance (ANOVA) is used to identify the parameters which are significantly influencing the process and contributes comparatively more towards occurrences of persisting defects [27,31].

\section{ANOVA analysis:}

The aim of the ANOVA tests is to look into the most significant parameter in green sand casting process that potentially affects the quality characteristics. The results of the ANOVA analysis of $\mathrm{S} / \mathrm{N}$ ratio correspond to the response; 
persisting defects in this study are reported in Tables 10 to 13 respectively. This analysis is performed to the level of significance $\alpha=0.05$, i.e. for a confidence level of $95 \%$. This analysis classifies the green sand casting process parameters in order to influence on various persisting defects. The ANOVA is carried out using the 'MINITAB 14' software. The results of Taguchi and ANOVA analysis are summarized in Table 12 given below. As mentioned in the summary; there is a close match between the results obtained by Taguchi approach and ANOVA analysis. For example, as mentioned in the table for ANOVA analysis of shrinkage porosity, carbon equivalent (CE) and percentage of steel scrap in the charge mix are found most influential parameters with percentage contributions of about $42.7 \%$ \& $22.3 \%$ respectively. Whereas from the Taguchi analysis and according to delta value corresponding rank of these parameters is $1 \& 2$. Same observations are made in case of other persisting defects that are sand inclusion, blowhole, and low hardness.

Table10 ANOVA results for signal-to-noise ratio for Shrinkage Porosity(Y1)

\begin{tabular}{l|l|l|l|l|l|l}
\hline Var & $\begin{array}{l}\text { Degre- } \\
\text { es of } \\
\text { Freed- } \\
\text { om }\end{array}$ & $\begin{array}{l}\text { Sum of } \\
\text { Squares } \\
\text { (SS) }\end{array}$ & $\begin{array}{l}\text { Mean } \\
\text { Square } \\
\text { (MS) }\end{array}$ & $\begin{array}{l}\text { F } \\
\text { ratio }\end{array}$ & P & \% Cont. \\
& & & & & \\
A & 1 & 28.665 & 28.6657 & 118.2 & 0 & $\mathbf{4 2 . 7 0 8 1 3}$ \\
B & 1 & 0.0786 & 0.0786 & 0.32 & 0.6 & 0.117104 \\
C & 1 & 0.2039 & 0.2039 & 0.84 & 0.411 & 0.303784 \\
D & 1 & 0.0201 & 0.0201 & 0.08 & 0.788 & 0.029946 \\
E & 1 & 14.967 & 14.9671 & 61.72 & 0.001 & $\mathbf{2 2 . 2 9 9 0 2}$ \\
F & 1 & 5.9795 & 5.9795 & 24.66 & 0.008 & $\mathbf{8 . 9 0 8 6 7 1}$ \\
G & 1 & 1.5724 & 1.5724 & 6.48 & 0.064 & 2.34267 \\
H & 2 & 0.4903 & 0.24515 & 1.01 & 0.441 & 0.730483 \\
J & 2 & 2.2582 & 1.1291 & 4.66 & 0.09 & 3.364422 \\
K & 2 & 0.2862 & 0.1431 & 0.59 & 0.596 & 0.4264 \\
L & 2 & 1.3704 & 0.6852 & 2.83 & 0.172 & 2.041716 \\
M & 2 & 0.4902 & 0.2451 & 1.01 & 0.441 & 0.730334 \\
N & 2 & 1.0407 & 0.52035 & 2.15 & 0.233 & 1.550507 \\
O & 2 & 0.9533 & 0.47665 & 1.97 & 0.254 & 1.420292 \\
P & 2 & 0.1769 & 0.08845 & 0.36 & 0.715 & 0.263558 \\
Q & 2 & 2.6462 & 1.3231 & 5.46 & 0.072 & 3.942491 \\
R & 2 & 1.4346 & 0.7173 & 2.96 & 0.163 & 2.137366 \\
S & 2 & 1.7334 & 0.8667 & 3.57 & 0.129 & 2.582539 \\
T & 2 & 1.7897 & 0.89485 & 3.69 & 0.124 & 2.666418 \\
Error & 4 & 0.9701 & 0.5463 & & & 1.16 \\
\hline Total & 35 & 67.127 & & & & $100 \%$ \\
\hline & & & & & & \\
\hline
\end{tabular}

Table 12. ANOVA results for signal-to-noise ratio for Sand Inclusion (Y3)

\begin{tabular}{c|c|c|c|c|c|c}
\hline Var & $\begin{array}{c}\text { Degre- } \\
\text { es of } \\
\text { Freed- } \\
\text { om } \\
\text { (DOF) }\end{array}$ & $\begin{array}{c}\text { Sum of } \\
\text { Squares } \\
\text { (SS) }\end{array}$ & $\begin{array}{c}\text { Mean } \\
\text { Square } \\
\text { (MS) }\end{array}$ & $\begin{array}{c}\text { F } \\
\text { ratio }\end{array}$ & P & \% Cont. \\
\hline A & 1 & 0.1388 & 0.1388 & 0.52 & 0.512 & 0.267954 \\
B & 1 & 0.8176 & 0.8176 & 3.05 & 0.156 & 1.578381 \\
C & 1 & 0.7958 & 0.7958 & 2.97 & 0.16 & 1.536296 \\
D & 1 & 0.8031 & 0.8031 & 3 & 0.158 & 1.550389 \\
E & 1 & 10.5693 & 10.5693 & 39.45 & 0.003 & $\mathbf{2 0 . 4 0 4 0 9}$ \\
F & 1 & 0.0652 & 0.0652 & 0.24 & 0.648 & 0.125869 \\
G & 1 & 0.1428 & 0.1428 & 0.53 & 0.506 & 0.275676 \\
H & 2 & 1.3906 & 0.6953 & 2.6 & 0.189 & 2.684561 \\
J & 2 & 0.1267 & 0.0634 & 0.24 & 0.8 & 0.244595 \\
K & 2 & 3.154 & 1.577 & 5.89 & 0.064 & 6.088815 \\
\hline
\end{tabular}

\section{Summary of Taguchi and ANOVA analysis}

The results obtained from Taguchi and ANOVA analysis are summarized in Table 14. As mentioned in the table, Taguchi and ANOVA results are verified for the group of parameters

\begin{tabular}{c|c|c|c|c|c|c}
\hline $\mathrm{L}$ & 2 & 14.5971 & 7.2986 & 27.24 & 0.005 & $\mathbf{2 8 . 1 7 9 7 8}$ \\
$\mathrm{M}$ & 2 & 0.0191 & 0.0095 & 0.04 & 0.965 & 0.036873 \\
$\mathrm{~N}$ & 2 & 5.3063 & 2.6532 & 9.9 & 0.028 & $\mathbf{1 0 . 2 4 3 8 4}$ \\
$\mathrm{O}$ & 2 & 5.128 & 2.564 & 9.57 & 0.03 & $\mathbf{9 . 8 9 9 6 3 3}$ \\
$\mathrm{P}$ & 2 & 0.701 & 0.3505 & 1.31 & 0.365 & 1.353284 \\
$\mathrm{Q}$ & 2 & 2.204 & 1.102 & 4.11 & 0.107 & 4.254834 \\
$\mathrm{R}$ & 2 & 1.6486 & 0.8243 & 3.08 & 0.155 & 3.182632 \\
$\mathrm{~S}$ & 2 & 2.5304 & 1.2652 & 4.72 & 0.089 & 4.884952 \\
$\mathrm{~T}$ & 2 & 0.5897 & 0.2949 & 1.1 & 0.416 & 1.13841 \\
Error & 4 & 1.0717 & 1.0717 & & & 2.07 \\
\hline Total & 35 & 51.7999 & & & & 97.93 \\
\hline
\end{tabular}

Table11. ANOVA results for signal-to-noise ratio for Blowhole (Y2)

\begin{tabular}{l|l|l|l|l|l|l}
\hline Var & $\begin{array}{c}\text { Degrees } \\
\text { of } \\
\text { Freedom } \\
\text { (DOF) }\end{array}$ & $\begin{array}{c}\text { Sum of } \\
\text { Square } \\
\text { s (SS) }\end{array}$ & $\begin{array}{c}\text { Mean } \\
\text { Square } \\
\text { (MS) }\end{array}$ & $\begin{array}{c}\text { F } \\
\text { ratio }\end{array}$ & P & Cont. \\
\hline A & 1 & 0.2899 & 0.2899 & 0.67 & 0.46 & 0.15975 \\
B & 1 & 0.2329 & 0.2329 & 0.54 & 0.504 & 0.12834 \\
C & 1 & 9.9382 & 9.9382 & 22.92 & 0.009 & $\mathbf{5 . 4 7 6 4 6 4}$ \\
D & 1 & 6.0333 & 6.0333 & 13.91 & 0.02 & $\mathbf{3 . 3 2 4 6 6 2}$ \\
E & 1 & 1.4696 & 1.4696 & 3.39 & 0.139 & 0.809826 \\
F & 1 & 2.2046 & 2.2046 & 5.08 & 0.087 & 1.214849 \\
G & 1 & 81.8304 & 81.8304 & 188.6 & 0 & $\mathbf{4 5 . 0 9 2 8}$ \\
& 2 & 14.2898 & 7.1449 & 16.47 & 0.012 & $\mathbf{7 . 8 7 4 4 2 2}$ \\
H & 2 & 20.0281 & 10.014 & 23.09 & 0.006 & $\mathbf{1 1 . 0 3 6 5 2}$ \\
J & 2 & 1.0129 & 0.5065 & 1.17 & 0.399 & 0.558161 \\
K & 2 & 2.8563 & 1.4281 & 3.29 & 0.143 & 1.57397 \\
L & 2 & 0.1056 & 0.0528 & 0.12 & 0.889 & 0.058191 \\
M & 2 & 6.9267 & 3.4634 & 7.99 & 0.04 & 3.816971 \\
N & 2 & 2.0422 & 1.0211 & 2.35 & 0.211 & 1.125358 \\
O & 2 & 2.3742 & 1.1871 & 2.74 & 0.178 & 1.308307 \\
P & 2 & 5.3887 & 2.6943 & 6.21 & 0.059 & 2.969454 \\
Q & 2 & 0.0083 & 0.0042 & 0.01 & 0.99 & 0.004574 \\
R & 2 & 7.4392 & 3.7196 & 8.58 & 0.036 & 4.099386 \\
S & 2 & 15.2656 & 7.6328 & 17.6 & 0.01 & 8.412138 \\
T & 2 & 1.7348 & 0.4337 & & & 1.30 \\
Err. & 4 & 181.4711 & & & & $100 \%$ \\
\hline Total & 35 & \multicolumn{5}{|l}{}
\end{tabular}

Table 13. ANOVA results for signal-to-noise ratio for Low Hardness (Y4)

\begin{tabular}{|c|c|c|c|c|c|c|}
\hline Var & $\begin{array}{l}\text { Degrees } \\
\text { of } \\
\text { Freedom } \\
\text { (DOF) }\end{array}$ & $\begin{array}{l}\text { Sum of } \\
\text { Squares } \\
\text { (SS) }\end{array}$ & $\begin{array}{l}\text { Mean } \\
\text { Square } \\
\text { (MS) }\end{array}$ & $\begin{array}{l}\mathbf{F} \\
\text { ratio }\end{array}$ & $\mathbf{P}$ & $\%$ Cont. \\
\hline $\mathrm{A}$ & 1 & 0.1229 & 0.1229 & 0.66 & 0.462 & 0.090509 \\
\hline B & 1 & 0.992 & 0.992 & 5.34 & 0.082 & 0.730551 \\
\hline $\mathrm{C}$ & 1 & 3.3011 & 3.3011 & 17.75 & 0.014 & 2.431069 \\
\hline D & 1 & 3.4386 & 3.4386 & 18.49 & 0.013 & 2.53233 \\
\hline $\mathrm{E}$ & 1 & 0.0086 & 0.0086 & 0.05 & 0.84 & 0.006333 \\
\hline $\mathrm{F}$ & 1 & 0.0004 & 0.0004 & 0 & 0.966 & 0.000295 \\
\hline G & 1 & 2.0847 & 2.0847 & 11.21 & 0.029 & 1.535261 \\
\hline $\mathrm{H}$ & 2 & 1.7569 & 0.8784 & 4.72 & 0.088 & 1.293855 \\
\hline $\mathrm{J}$ & 2 & 0.8577 & 0.4289 & 2.31 & 0.216 & 0.631646 \\
\hline K & 2 & 0.1732 & 0.0866 & 0.47 & 0.658 & 0.127552 \\
\hline $\mathrm{L}$ & 2 & 15.9915 & 7.9957 & 43 & 0.002 & 11.77681 \\
\hline M & 2 & 0.1376 & 0.0688 & 0.37 & 0.712 & 0.101334 \\
\hline $\mathrm{N}$ & 2 & 0.0484 & 0.0242 & 0.13 & 0.881 & 0.035644 \\
\hline $\mathrm{O}$ & 2 & 0.0926 & 0.0463 & 0.25 & 0.791 & 0.068195 \\
\hline $\mathrm{P}$ & 2 & 5.2142 & 2.6071 & 14.02 & 0.016 & 3.839956 \\
\hline Q & 2 & 7.9504 & 3.9752 & 21.38 & 0.007 & 5.855009 \\
\hline $\mathrm{R}$ & 2 & 0.9324 & 0.4662 & 2.51 & 0.197 & 0.686659 \\
\hline S & 2 & 1.1164 & 0.5582 & 3 & 0.16 & 0.822164 \\
\hline $\mathrm{T}$ & 2 & 90.8246 & 45.4123 & 244.23 & 0 & 66.88706 \\
\hline Errorr & 4 & 0.7438 & 0.1859 & & & 0.55 \\
\hline Total & 35 & 135.788 & & & & 99.45 \\
\hline
\end{tabular}

which are associated with a process or operation responsible for the occurrences of respective persisting defects. The major findings in this summary are as given below,

1. Taguchi results and ANOVA results are having close matches. 
2. Delta value and corresponding rank in the Taguchi response table for Significant parameters identified in the ANOVA analysis $(\mathrm{P}<0.05)$ is having good agreement with each other.

3. Percentage contribution made by group of parameters that are associated with particular process and in turn responsible for persisting defects is in the range of $60-75 \%$, as compared to contributions made by other process parameters consider for experimentation.

4. Contributions made by other parameters are in the range of $25-40 \%$

Table 14. Summary of Taguchi and ANOVA analysis

\begin{tabular}{|c|c|c|c|c|c|c|c|c|}
\hline $\begin{array}{l}\text { Quality } \\
\text { Characteristics }\end{array}$ & $\begin{array}{ll}\text { Selected } & \text { Process } \\
\text { Parameters } & \end{array}$ & Designation & $\begin{array}{l}P \quad \text { in } \\
\text { ANOVA } \\
\text { Table }\end{array}$ & $\%$ Contt & Remarks & $\begin{array}{l}\text { Delta Value } \\
\text { (Taguchi } \\
\text { Rank) }\end{array}$ & $\begin{array}{l}\text { Optimal } \\
\text { setting }\end{array}$ & $\begin{array}{l}\text { Optimal } \\
\text { Level }\end{array}$ \\
\hline \multirow[t]{7}{*}{$\begin{array}{l}\text { Shrinkage } \\
\text { Porosity }\end{array}$} & $\begin{array}{ll}\text { Carbon } & \text { Equivalent } \\
\text { Value (nu) } & \end{array}$ & A & 0 & 42.70813 & Significant & $1.785 \quad(1)$ & 4.0 & Level 2 \\
\hline & Carbon $(\%)$ & B & 0.6 & 0.117104 & $\ldots$. & $0.093(18)$ & 3.4 & Level 2 \\
\hline & $\begin{array}{l}\text { Pouring Temperature } \\
\left({ }^{\circ} \mathrm{C}\right)\end{array}$ & C & 0.411 & 0.303784 & $\ldots$ & 0.151 (17) & 1400 & Level 1 \\
\hline & Inoculants Qty (\%) & $\mathrm{D}$ & 0.788 & 0.029946 & & 0.047 (19) & 0.15 & Level 1 \\
\hline & $\begin{array}{l}\text { Steel Scrap in Charge } \\
\operatorname{mix}(\%)\end{array}$ & E & 0.001 & 22.29902 & Significant & 1.290 & 30 & Level 1 \\
\hline & $\begin{array}{l}\text { Phosphorus content } \\
(\%)\end{array}$ & $\mathrm{F}$ & 0.008 & 8.908671 & Significant & 0.815 & 0.08 & Level 1 \\
\hline & \multicolumn{8}{|c|}{ Total Contribution $(\%)=74.36 \%$} \\
\hline \multirow[t]{6}{*}{ Blowholes } & Binder $(\%)$ & $\mathrm{G}$ & 0 & 45.0928 & Significant & $3.01536(1)$ & 1.8 & Level 1 \\
\hline & Moisture (\%) & $\mathrm{H}$ & 0.012 & 7.874422 & Significant & $1.50427(4)$ & 4.0 & Level 1 \\
\hline & Permeability & $\mathrm{J}$ & 0.006 & 11.03652 & Significant & $1.80212(2)$ & 150 & Level 3 \\
\hline & Pouring Temp. $\left({ }^{0} \mathrm{C}\right)$ & $\mathrm{C}$ & 0.009 & 5.476464 & Significant & $1.05083(7)$ & 1400 & Level 1 \\
\hline & Sulphur content (\%) & $\mathrm{K}$ & 0.399 & 0.558161 & $\ldots \ldots$ & $\begin{array}{l}0.39845 \\
(15)\end{array}$ & 0.09 & Level 3 \\
\hline & \multicolumn{8}{|c|}{ Total Contribution $(\%)=70.03 \%$} \\
\hline \multirow[t]{8}{*}{ Sand Inclusion } & $\begin{array}{l}\text { Green Compression } \\
\text { Strength }(\mathrm{Kg} / \mathrm{Cm} 2)\end{array}$ & $\mathrm{L}$ & 0.005 & 28.17978 & Significant & $1.5589(1)$ & 1.8 & Level 3 \\
\hline & Moisture (\%) & $\mathrm{H}$ & 0.189 & 2.684561 & $\ldots$ & $0.46967(9)$ & 4.6 & Level 2 \\
\hline & Compatibility (\%) & $\mathrm{M}$ & 0.965 & 0.730334 & $\ldots$ & $0.04971(19)$ & 43 & Level 2 \\
\hline & $\begin{array}{ll}\text { Return } & \text { Sand } \\
\text { Temp. }\left({ }^{0} \mathrm{C}\right) & \end{array}$ & $\mathrm{N}$ & 0.028 & 10.24384 & Significant & $0.94022(3)$ & 30 & Level 1 \\
\hline & $\begin{array}{l}\text { Squeezing } \\
\text { (bar) }\end{array}$ & $\mathrm{O}$ & 0.003 & 9.899633 & Significant & $0.81297(4)$ & 100 & Level 2 \\
\hline & $\begin{array}{l}\text { Mould Hardness } \\
\text { Horizontal (nu) }\end{array}$ & $\mathrm{P}$ & 0.365 & 1.353284 & $\ldots$. & $0.31377(10)$ & 90 & Level 3 \\
\hline & $\begin{array}{l}\text { Mould Hardness } \\
\text { Vertical (nu) }\end{array}$ & Q & 0.107 & 4.254834 & $\ldots$ & $0.58588(7)$ & 70 & Level 1 \\
\hline & \multicolumn{8}{|c|}{ Total Contribution $(\%)=57.34 \%$} \\
\hline \multirow[t]{5}{*}{ Low Hardness } & $\begin{array}{l}\text { Carbon Equivalent } \\
\text { Value (CE) }\end{array}$ & A & & & & $0.117(15)$ & & \\
\hline & $\begin{array}{l}\text { Knockout } \quad \text { Time } \\
\text { (Minute) }\end{array}$ & $\mathrm{S}$ & 0.16 & 0.822164 & $\ldots$ & 0.405 (9) & 30 & Level 1 \\
\hline & Inoculants Qty (\%) & $\mathrm{D}$ & 0.013 & 2.53233 & Significant & $0.6189(5)$ & 0.15 & Level 1 \\
\hline & Manganese (\%) & $\mathrm{T}$ & 0 & 66.88706 & Significant & $3.89(1)$ & 0.8 & Level 3 \\
\hline & \multicolumn{8}{|c|}{ Total Contribution $(\%)=70.33 \%$} \\
\hline
\end{tabular}

\section{Confirmation Experiments} step for completion of any DOE study. Before accepting determined, one of the following two possibilities exists: predicted optimum condition, it is essential that the predicted

performance at the optimum be confirmed by running a number 1. The prescribed combination of parameters at optimal of samples in that condition. To confirm predicted performance, levels is identical to one of those in the experiments mean (average) of the actual test results is compared with the conducted.

confidence interval calculated for the expected performance 2. The prescribed combination of parameters at optimal $[7,30]$.

\subsection{Estimation of mean}

levels is not obtained in the experiments conducted with chosen orthogonal array.

In the present work, the second situation exists, and hence, to estimate the mean for that treatment condition is to 
average all the results for the trials which are set at levels those are optimal levels of parameters. The estimation of mean for casting defect, shrinkage porosity is achieved by the following equation.

$\mu($ Shrinkage Porosity $)=T+(\overline{A 2}-T)+(\overline{B 2}-T)+$ $(\overline{C 1}-T)+(\overline{D 1}-T)+(\overline{E 1}-T)+(\overline{F 1}-T)+(\overline{G 1}-T)$ $+(\overline{H 1}-T)+(\overline{J 3}-T)+(\overline{K 1}-T)+(\overline{L 3}-T)+(\overline{M 1}-T)$

$+(\overline{N 3}-T)+(\overline{O 2}-T)+(\overline{P 3}-T)+(\overline{Q 1}-T)+(\overline{R 2}-T)$ $+(\overline{S 3}-T)+(\overline{T 3}-T)$

Where, $\mu$ is the mean of casting defects at optimal level of parameters. ' $T$ ' is the average value of shrinkage porosity defect and $\overline{A 2}, \overline{B 2}, \overline{C 1}, \ldots \ldots . \overline{T 3}$ are the average of shrinkage porosity defect at optimal level of parameters .The mean (optimal value) for a selected trial condition for parameters of the level's mentioned in the above equation is

\section{$\boldsymbol{\mu}$ (Shrinkage Porosity) $=1.687 \%$.}

Similarly, mean $(\mu)$ for other defects are calculated as given below,

$$
\begin{aligned}
& \mu(\text { Blow Hole })=T+(\overline{A 1}-T)+(\overline{B 2}-T)+(\overline{C 1}-T)+ \\
& (\overline{D 2}-T)+(\overline{E 1}-T)+(\overline{F 2}-T)+(\overline{G 1}-T)+(\overline{H 1}-T) \\
& +(\overline{J 3}-T)+(\overline{K 3}-T)+(\overline{L 3}-T)+(\overline{M 2}-T)+(\overline{N 1}-T) \\
& +(\overline{O 1}-T)+(\overline{P 3}-T)+(\overline{Q 1}-T)+(\overline{R 1}-T)+(\overline{S 1}-T) \\
& +(\overline{T 1}-T)
\end{aligned}
$$

$\boldsymbol{\mu}($ Blowhole $)=0.1931 \%$.

$$
\begin{aligned}
& \mu(\text { Sand } \text { Inclusion })=T+(\overline{A 2}-T)+(\overline{B 1}-T)+(\overline{C 1}-T)+ \\
& (\overline{D 1}-T)+(\overline{E 1}-T)+(\overline{F 2}-T)+(\overline{G 1}-T)+(\overline{H 2}-T)+ \\
& (\overline{J 2}-T)+(\overline{K 3}-T)+(\overline{L 3}-T)+(\overline{M 2}-T)+(\overline{N 1}-T)+ \\
& (\overline{O 2}-T)+(\overline{P 3}-T)+(\overline{Q 1}-T)+(\overline{R 3}-T)+(\overline{S 2}-T) \\
& +(\overline{T 2}-T)
\end{aligned}
$$

$\boldsymbol{\mu}($ Sand Inclusion $)=0.465 \%$

$$
\begin{aligned}
& \mu(\text { Low Hardness })=T+(\overline{A 1}-T)+(\overline{B 2}-T)+(\overline{C 1}-T) \\
& +(\overline{D 1}-T)+(\overline{E 1}-T)+(\overline{F 1}-T)+(\overline{G 2}-T)+(\overline{H 3}-T) \\
& +(\overline{J 1}-T)+(\overline{K 2}-T)+(\overline{L 3}-T)+(\overline{M 3}-T)+(\overline{N 2}-T) \\
& +(\overline{O 2}-T)+(\overline{P 3}-T)+(\overline{Q 3}-T)+(\overline{R 3}-T)+(\overline{S 1}-T) \\
& +(\overline{T 3}-T)
\end{aligned}
$$

\footnotetext{
$\boldsymbol{\mu}($ Low Hardness $)=0.04 \%$
}

\subsection{Confidence interval around mean}

The optimum levels of process parameters have already been obtained. The estimate of the mean $(\mu)$ is only a point estimate based on the average of the output values obtained as a result of experiments conducted. Statistically, this provides a $50 \%$ chance of the true average being greater than $\mu$ and a $50 \%$ chance of the true average being less than $\mu$. The confidence level is the maximum and minimum value between which the true average should fall at some stated level. The $95 \%$ confidence interval of confirmation experiments (CICE) is calculated by using the following equations

$$
\mathrm{CICE}=\left[F(\alpha, 1, v e) V e\left[\left(1 / \eta_{\mathrm{eff}}\right)+(1 / r)\right]\right]^{1 / 2}
$$

Where $\alpha$ is the level of risk, $V e$ is the error variance, ve is the degrees of freedom for the error. neff is the effective number of replications and $r$ is the number of test trials in confirmatory experiments.

$\eta_{\text {eff }}=N /(1+$ total degree of freedom associated in the estimation of mean)

$N=$ total experiments $=36$

$\eta \mathrm{eff}=36 /(1+31)=36 /(1+31)=1.125$

$F(\alpha, 1, v e)=F(0.05,1,4)=7.7086($ tabulated $)$

$\alpha=1$ - Confidence limit $(95 \%)=0.05$

$\mathrm{Ve}$ is the error variance $=0.02964$

$v e$ is the degrees of freedom for the error $=4$

$V e \& v e$ is calculated from ANOVA analysis for casting defect output i.e. shrinkage porosity

$\mathrm{CI}=\left[7.7086 \mathrm{x} 0.02964[1 / 1.125+1 / 10]^{1 / 2}\right.$

$\mathrm{CI}= \pm 0.4752$

Predicted optimal range (for a confirmation run with 10 experiments) at $95 \%$ interval for respective casting defects is

$[\mu-\mathrm{CI}]<\mu<[\mu+\mathrm{CI}]$

Therefore, For shrinkage porosity,

$[1.687-0.4752]<1.687<[1.687+0.4752]$

\section{$1.2118<1.1687<2.1622$}

Similarly, CI is calculated for other persisting defects that are blowhole, sand inclusion, and low hardness to predict optimal range at $95 \%$ and values obtained is as given below,

For blowhole,

$\mu($ Blowhole $)=0.1931, C I= \pm 0.2647$

$[0.1931-0.2647]<0.1931<[0.1931+0.2647]$

$0.0716<0.1931<0.4578$

For sand inclusion,

$\mu($ Sand Inclusion $)=0.4655, C I= \pm 0.172$ 
$[0.4655-0.172]<0.4655<[0.4655+0.172]$

$0.293<0.4655<0.6375$

For Low Hardness,

$\mu($ Low Hardness $)=0.04, C I= \pm 0.0393$

$[0.04-0.0393]<0.04<[0.04+0.0393]$

$0.0007<0.04<0.0793$

\subsection{Results of confirmation run}

The objective of the confirmation run is to determine that the selected control parameter values at optimum level will produce better results than those produced in the first part of the experiment. The confirmation experiments are used for verification of the optimal levels suggested through screening experiments for selected potential parameters that will give desired output of the process. The confirming experiment is an important step to verify the experimental conclusions and is interpreted in this manner. If the average of the output of the confirmation run is within the range of the confidence limits, then the significant factors as well as the optimum levels for obtaining the desired results are properly chosen. If the average of the results of the confirmation experiment is not within the range of the CI, the parameters selected and/or levels to control the results for a desired value are incorrect or have excessive measurements, in this case further experimentation is required [29]. Ten confirmation experiments are conducted at the optimum settings of the process as shown in Table 15. The average of the results of confirmation experiments are 2.093 for shrinkage porosity which is less than $2.1622 \%$ (maximum of CI), 0.441 for blowhole which is less than $0.4578,0.62$ for sand inclusion which is less than 0.6375 and 0.072 for low hardness which is less than 0.0793Therefore, the selected parameters as well as their appropriate levels are significant enough to obtain the desired result.

Table 15. Results of confirmation run

\begin{tabular}{l|l|l|l|l}
\hline Experimentsat \\
$\begin{array}{l}\text { optimum level } \\
\text { of parameters } \\
\text { for respective } \\
\text { defects }\end{array}$ & $\begin{array}{l}\text { Shrinkage } \\
\text { Porosity }\end{array}$ & $\begin{array}{l}\text { Blow } \\
\text { Hole }\end{array}$ & $\begin{array}{l}\text { Sand } \\
\text { Inclusion }\end{array}$ & $\begin{array}{l}\text { Low } \\
\text { Hardness }\end{array}$ \\
\hline 1 & & & & \\
2 & 3.1 & 0.7 & 0.8 & 0.1 \\
3 & 2.26 & 0.58 & 0.6 & 0.1 \\
4 & 1.89 & 0.54 & 0.7 & 0.1 \\
5 & 2.38 & 0.6 & 1 & 0.17 \\
6 & 1.92 & 0.27 & 0.63 & 0.03 \\
7 & 1.9 & 0.3 & 0.48 & 0.05 \\
8 & 1.45 & 0.36 & 0.71 & 0.08 \\
9 & 1.88 & 0.48 & 0.46 & 0.04 \\
10 & 1.7 & 0.28 & 0.21 & 0.05 \\
\hline Average \% & 2.45 & 0.3 & 0.61 & 0 \\
\hline
\end{tabular}

\section{Conclusions}

In this work 19 process parameters associated with principal operations in green sand process are selected to determine optimal level for minimization of defects whch are more persisting in gray iron cast components. The defects which are found persisting in cylinder head castings produced in foundry in central India are shrinkage porosity, blowhole, sand inclusion and low hardness are the defects which are found persisting and contribute to productivity loss with increased production cost. These defects are analyzed by Taguchi method and ANOVA analysis. The important findings of this work are group of parameters are identified which are most influential in occurrences of respective persisting defects exposed in this study. The details about the study and findings are listed below.

1. The mixed orthogonal array of Taguchi is used to determine the optimal setting of the green sand casting process parameters in order to minimize the persisting defects in this process.

2. Based on the ANOVA analysis of $\mathrm{S} / \mathrm{N}$ ratio the most influential parameters are identified which are responsible for the occurrences of persisting defects in complex castings for example cylinder head cast component considered in this study.

3. Carbon equivalent (CE) value, percentage (\%) of steel scrap in charge mix and percentage $(\%)$ of phosphorus content in gray iron is found most influential parameters and having contribution of $42.70 \%, 22.3 \%$ and $8.90 \%$, respectively in the occurrence of shrinkage porosity defect. 4. In Persisting defect blowhole, percentage of binder (resin used in 2-part no bake core making system) is the most influential parameter followed by other parameters that are, permeability, percentage of moisture in green sand and pouring temperature. The contributions made by these parameters are $45.09 \%, 11.03 \%, 7.87 \%$ and $5.47 \%$ respectively.

5. Green compression strength of sand, return sand temperature and squeezing pressure on the sand during mould preparation are found most influential parameters and responsible for sand inclusion defects with a contribution of $28.17 \%, 10.24 \%$ and $9.89 \%$.

5. For the persisting defect low hardness in cast components, percentage of manganese in metal is the most influential parameter and contribution made is $66.88 \%$.

6. As presented in the Table 12, summary of Taguchi and ANOVA analysis, results obtained by Taguchi and ANOVA analysis are verified for the parameters selected on the basis of their association with respective persisting defects. It is observed from the summary that, group of selected parameters contributes in the range of about $60-75 \%$ (including significant parameters) in the occurrences of respective persisting defects.

7. In green sand casting process great number of parameters is involved, but if the defects are properly identified and associated processes or operation is correctly defined which is responsible for the occurrences of respective defects, defects can be minimized through proper setting of parameters. In the initial course of action only significant parameters can be targeted instead of changing setting of so many other parameters used in trial and error approach

8. The results of the confirmation test prove that the persisting defects found in gray iron castings produced in green sand casting process that are shrinkage porosity, blowhole, sand inclusion and low hardness consider in this work are minimized through the optimal combination of the green sand casting process parameters.

\section{Acknowledgements}

The study presented in this paper is carried out in a NECO industry located in Nagpur, central India, medium size 
foundry, producing automobiles castings mainly including cylinder heads ( 3 to 6 bore) and housings for domestic and global customers by a green sand casting process. The assistance of foundry experts in the process of selection of essential input parameters affecting persisting casting defects as well as in conducting and recording the experiments is highly acknowledged

This is an Open Access article distributed under the terms of the Creative Commons Attribution License

\section{References}

1. D. E. Krause, "Gray Iron-A Unique Engineering Material" Executive Director , 1940-1973(The Gray Iron Research Institute)Iron Casting Research Institute(1969).

2. T. N. Desai and Dr. R. L. Shrivastava, Six Sigma - A New Direction to Quality and Productivity Management, World Congress on Engineering and Computer Science 2008 WCECS 2008, October 22 - 24, 2008, San Francisco, USA(2008)

3. A. Kumarvadivel, U. Natrajan Optimization of Sand Casting Process Variables- A Process Window Approach .Int J Adv Manuf Technol 66:695-709 (2013).

4. R. L. Shrivastava , R. P. Mohanty \& R. R. Lakhe, Linkages between total quality management and organisational performance: an empirical study for Indian industry, Production Planning \& Control: The Management of Operations, 17:1, 13-30(2006).

5. R. Monroe, Porosity in Castings, AFS Transactions Paper 05245(04) (2005).

6. G.Taguchi, Introduction to Quality Engineering. Asian Productivity Organization, Tokyo, Japan(1986)

7. P. J. Ross, Taguchi techniques for quality engineering,2nd edn. McGraw Hill, New York(1996).

8. ASM International Committee, Metals handbook for castings.ASM International, 10th edn, vol 15. Materials Park, OH (1990).

9. A.Mandal, and P. Roy, Modeling the compressive strength of molasses-cement sand system using design of experiments and back propagation neural network. Journal of Materials Processing Technology, 180, 167-173(2006).

10. S. Guharaja, Noorul Haq, A. K. M. Karuppannan Optimization of green sand casting process parameters by using Taguchi's method. Int J Adv Manuf Technol 30:1040-1048(2006).

11. D.B. Karunakar and G. L. and Datta, Prevention of defects using back propagation neural Networks. International Journal of Advanced Manufacturing Technology, 39, 1111-1124(2008).

12. M. B. Parappagoudar et al. Neural Forward and reverse mappings in green sand mould system using neural networks. Applied Soft Computing 8:239-260(2008).

13. A. Noorul Haq, S. Guharaja, K.M. Karuppanan, Parameter optimization of $\mathrm{CO} 2$ casting process by using Taguchi method. Int J Interact Des Manuf 3:41-50(2006).

14. B. Senthilkumar, S.G. Ponnambalam, Process factor optimization for controlling pull-down defects in iron castings,Journal of Materials Processing Technology 209:554-560.(2009).

15. Sushil Kumar, P. S. Satsangi , D. R. Prajapati, Optimization of green sand casting process parameters of a foundry by using Taguchi's method. International Journal of Advanced Manufacturing Technology 55:23-34(2011).

16. Charnnarong Saikaew, Sermsak Wiengwiset, Optimization of molding sand composition for quality improvement of iron castings, Applied Clay Science 67-68:26-31(2012).

\section{Nomenclature}

ANOVA Analysis of variance

Cont $\%$ Percentage Contribution made by individual parameter

CE Carbon equivalent

DOE Design of Experiments

DOF Degree of freedom

MS Mean squares

OA Orthogonal array

S/N Signal-to-noise ratio

SS Sum of squares

$\mathrm{T} \quad$ Average value of casting defects at different levels

yi Response value of observation in ith test
17. R. A.Upadhye and I. P. Keswani, Optimization of Sand Casting Process Parameter Using Taguchi Method in Foundry. International Journal of Engineering Research and Technology, 1(7), 1-11(2012).

18. U. A. Dabade and R.C. Bhedasgaonkar, Casting Defect Analysis using Design of Experiments (DoE) andComputer Aided Casting Simulation Technique,Procedia CIRP Science Direct 00:000000(2013).

19. A. Kumaravadivel et.al. Optimization of sand-casting process variables - a process window approach, International Journal of Advanced Manufacturing Technology 66:695-709(2013).

20. Ganesh G. Patil, Dr. K. H. Inamdar, Optimization of Casting Process Parameters using Taguchi Method, International Journal of Engineering Development and ResearchVol.1:Issue1(2014).

21. Manjunath Patel G, Prasad Krishna and Mahesh B. Parappagoudar, Optimization of squeeze cast process parameters using Taguchi and grey relational analysis,Procidia Technology 14:157-164(2014).

22. S.K.Tiwari,R.K.Singh,S.C.Srivastava, ,Optimisation of green sand casting processparameters for enhancing quality of mild steel castings', Int. J. Productivity and Quality Management, Vol. 17, No. 2, pp.127-141(2016).

23. A. Johnson Santhosh and A. R. Lakshmanan, Investigation of ductile iron casting process parameters using Taguchi approach and response surface methodology, China Foundry 13:352-360(2016).

24. Madhav S. Phadke, Quality Engineering Using Robust Design, Pearson Publication, India(2009).

25. Taguchi, G., Introduction to Quality Engineering. Asian Productivity Organization, Tokyo, Japan (1986).

26. D.C. Montgomery ,Design and analysis of experiments, 4th edn. Wiley, New York,(1997).

27. T. P. Enright, B. Prince, Offline quality control parameter estimation and experimental design with the Taguchi method. AFS Transactions. pp. 393-400(1983)

28. Oussama Zerti, Mohamed Athmane Yallese, \& Riad Khettabilet.al. ,Design optimization for minimum technological parameters when dry turning of AISI D3 steel using Taguchi method. International Journal of Advanced Manufacturing Technology(2016).

29. M. Lipinski, W .Schaefer, S. Andersen, Modeling of combined heat and fluid flow for determination of filling sequence for real complex shaped castings. In: Modeling of casting, welding and advanced solidification processes. TMS, Warrendale, PA, pp 185211(1991).

30. Ching-Been Yang et. al., Single and multiobjective optimization of Inconel 718 nickel-based superalloy in the wire electrical discharge machining, Int J Adv Manuf Technol Volume 93, pp 30753084(2017).

31. M. Nataraj and K. Balasubramanian, Parametric optimization of CNC turning process for hybrid metal matrix composite, Int J Adv Manuf Technol, Volume 93, pp 215-224(2017). 